\title{
Mathematics or Mathematics for Engineering?
}

\author{
Paula Carvalho \\ Department of Mathematics \\ University of Aveiro \\ Aveiro, Portugal \\ paula.carvalho@ua.pt
}

\author{
Paula Oliveira \\ Department of Mathematics \\ University of Aveiro \\ Aveiro, Portugal \\ paula.oliveira@ua.pt
}

\begin{abstract}
It is undeniable that mathematics is indispensable in education and practice of Engineering in all areas. In this paper we discuss the use of some active learning strategies on calculus applied in the first and second years of Engineering degrees. Among other experiences we report a flipped classroom experience, autonomous study and self evaluation using dedicated technological platforms and also active learning by solving proposed challenges outside the classroom where group and team work is encouraged in order to improve writing skills and the ability to communicate in mathematics.
\end{abstract}

Index Terms - calculus, Engineering, university teacher education, active learning, personal relationship.

\section{INTRODUCTION}

In several science and technology curricula, Calculus is among the first courses to be taught. Calculus is considered one of the most important courses in Engineering in the first years, allowing students to subsequently study and model real problems and applications to their professional lives. Despite this, Calculus teachers often give more importance to the application of techniques and the manipulation of formulae, rather than the acquisition of notions that are relevant to the practice of Engineering. In some known cases this can be a cause for the students failure on Calculus and perhaps for the abandonment of their professional ambitions.

Engineering courses are often organised into two main groups: general science courses, where we may include mathematics, chemistry and physics, and technical courses specific to each branch of Engineering.

In the first years of their degrees students seem to be unable to understand where and when they will practically apply the mathematics they are learning. On the other hand, they may find it challenging to recognise and apply this knowledge in later courses.

As [1] concludes, mathematics should be embedded with the Engineering principles being taught. This is dangerous when mathematics becomes isolated from its use in Engineering, and the opportunity to foster a perception of its use-value in the wider sense is lost.

In recent years, with the fast development of technology in general and particularly in education, educators and researchers increasingly highlight the potential merits of using educational technology to improve Science, Technology, Engineering and Mathematics (STEM) learning outcomes. We believe that on-line learning environments and the way learning resources are delivered may be crucial in achieving better learning outcomes.

In fact, digital technologies provide new possibilities for mathematics visualization, for interacting with mathematical concepts and for understanding mathematics in the context of the curricula.

God has made science such that we can repeat our designs once we have computed and tested the math. Computer science now makes the math resolutions much quicker and simpler but it is necessary to understand the basic principals so that you can affirm the computer results. Trial and error in engineering is no longer an acceptable means. It's important to have a good foundation in mathematics as an engineering [11].

Keeping this in mind, it is appropriate to ask: what mathematics should be taught in Engineering early courses? Would there be a difference in the mathematics offered to different Engineering courses? Can technology be used advantageously in the early years of university teaching?

Some studies on the transfer of specific mathematics skills and mathematical knowledge by university students into new subject areas, including science and Engineering can be found in the literature (for instance in [1]) All of them mention that students can experience problems in transferring these core skills to different contexts. So, in general, mathematics is not just a tool that can be picked up and applied in other subjects without adjustment, modeling, and even sometimes rebuilding ones mathematical knowledge.

However, one can also ask whether other variables such as different methodologies, teacher's behavior and teacher/student relationships can affect the performance of students learning. We can find also some reports on more academically engaging pedagogies, such as whole-class discussion, students explaining their thinking, and working together in groups, which are related to calculus persistence among students (see [2]).

In this paper we discuss the use of some active learning strategies on calculus applied in the first and second years of Engineering degrees. Among other experiences we report a flipped classroom experience, autonomous study and self evaluation using dedicated technological platforms and also active learning by solving proposed challenges outside the classroom, where group and team work is encouraged in order to improve writing skills and the ability to communicate in mathematics. 


\section{ACtive LEARNING IN MATHEMATICAL CLASSES}

\section{A. Flipped classroom}

In a flipped classroom, students prepare themselves at home, watching video lectures or studying lecture notes previously given by their teachers or available on line, as is current nowadays and classroom time is dedicated to learning activities that require students to engage concepts at a higher level in a group setting and with an instructor at hand to answer questions, give feedback, and prompt re-examination of key ideas. [12]

In Calculus II, a curricular unit for the 1st year on Engineering and Sciences degrees, we experienced flipping some classes on chosen topics, so that students turned into active participants. We've done the experience for two years and, based on students opinion, it is something worth continuing.

To support autonomous study a detailed text was available on moodle as well as a platform with worked-out exercises, described in section IV.

During the class, students working in groups of 4 or 5 elements, solved some problems/exercises on the subject studied at home, and the teacher clarified the doubts raised by each group.

In the last 20 minutes of the class each student had to answer individually to a question (class question) on the subject of that specific class. The grades obtained in these class questions could be used to improve the final score on the course.

\section{B. Challenges}

As referred in [10], there are some fundamental areas that most engineers are likely to encounter in their careers. Calculus is certainly one of them; any problem that has variables that are changing with respect to one another, like position and time, or voltage and time, or position and voltage involves calculus.[...] But regardless of the extent to which engineers use a particular type of mathematics in their daily work, all of their thinking towards solving problems is informed by the principles and logic of these foundations of mathematics.

In recent years there has been an increasing emphasis on active learning focusing the learning activity in the student. Problem solving, rather than only straightforward applications of algorithms, has become an important objective in the learning of mathematics. Calculus III is also a one semester curricular unit for 2nd year students on Engineering and Sciences degrees. Along the semester Calculus III students are faced with a small set of issues, which we call challenges. This proposal aims to encourage confidence in learning mathematics, to perceive usefulness of mathematics, to control attitude towards success in mathematics, to promote discussion between peers and group work, to use the recommended bibliography or be able to find another, as well as to stimulate the habit of doing and writing mathematics. These challenges are also an indicator of the level of mathematical thinking of the students.

Often, the challenges proposed are related with real problems, such as applications to simple technical ones, but some of them are simply challenging problems on their own. Students have, in general, one or two weeks to solve them. In Example 1 we describe a problem about a cup of coffee that forces students to think and find the better way of solving it using computational tools. The problem is of very simple nature but the choice of the coordinate system is fundamental. We remark that if the cup has the shape of a conic or cylindrical surface the problem is trivial.

Example 1: Imagine you are drinking a coffee. The cup is half of a spherical surface that can be described by the equation $x^{2}+y^{2}+(z-4)^{2}=16$ with a spherical cap removed in such a way that the basis of the cup is a disk of radius 2. Suppose you stop drinking when you start to see the bottom of the cup. See figure 1 for an illustration. Use triple integration to compute the volume of remaining coffee. Choose the more convenient coordinate system.

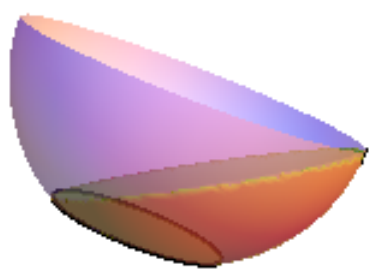

Fig. 1. Coffee cup: Example 1

The solution is given by

$$
2 \int_{4-2 \sqrt{3}}^{4} \int_{4+\sqrt{3}(z-4)}^{\sqrt{16-(z-4)^{2}}} \int_{0}^{\sqrt{16-y^{2}-(z-4)^{2}}} d x d y d z .
$$

in Cartesian coordinates. One can use a computational system in a long time-consuming process to compute this integral (try it!), but there is a surprisingly simple way to perform it by putting the cup on a table, looking to the shape the liquid then takes and using spherical coordinates:

$$
\begin{array}{r}
\int_{0}^{2 \pi}\left(\int_{\frac{2 \pi}{3}}^{\pi}\left(\int_{-\frac{2}{\cos \phi}}^{4} \rho^{2} \sin \phi d \rho\right) d \phi\right) d \theta \\
-\int_{0}^{2 \pi}\left(\int_{\frac{5 \pi}{6}}^{\pi}\left(\int_{-\frac{2 \sqrt{3}}{\cos \phi}}^{4} \rho^{2} \sin \phi d \rho\right) d \phi\right) d \theta .
\end{array}
$$

The solution is 38.4402 , obtained in less than one second. As it is expected, however, the majority of students chose cylindrical coordinates. In this case, the integral to be computed is:

$$
\begin{array}{r}
\int_{0}^{2 \pi} \int_{0-\sqrt{16-(r \cos \theta)^{2}-\left(1+\frac{\sqrt{3}}{2} r \sin \theta\right)^{2}}}^{\sqrt{12}}\left(\frac{\sqrt{3}}{2} r\right) d z d r d \theta \\
-\int_{0}^{2 \pi} \int_{0}^{2} \int_{4-\sqrt{16-r^{2}}}^{4-2 \sqrt{3}} r d z d r d \theta
\end{array}
$$

which is again too difficult to compute (the first one) even with a computer.

In this case, students are encouraged to use computational tools but they find severe difficulties in obtaining the solution 
except if they are able to choose and work with the right system of coordinates, which is the less obvious.

\section{TECHNOLOGY AND MATHEMATICS}

It is undeniable that mathematics is indispensable in education and practice of Engineering in all areas. A solid grounding in Mathematics is a part of every undergraduate Engineering degree and Calculus (although it's not the only one) is a branch of mathematics that typically concerns the study of limits, continuity and differentiability of functions depending on a single variable in the first semester of the first year, and several variables in the second semester and/or in the second year. This subject is very conceptual and often a serious obstacle to a good performance in elementary calculus. Commonly, students are able to compute the limit values by applying standard procedures but are unable to use the limit concept in solving related problems. Besides, as there are several accessible computer systems to compute limits of functions, students tend to use them with confidence and without critical spirit. A short experience was made in 2015 with mechanical Engineering students in second year Calculus. The aim of this experience was to find out whether students were able to use their knowledge, skills and critical performance to prevent an error given by a very popular on-line computer system: Wolfram Alpha [3]. See Figure 2.

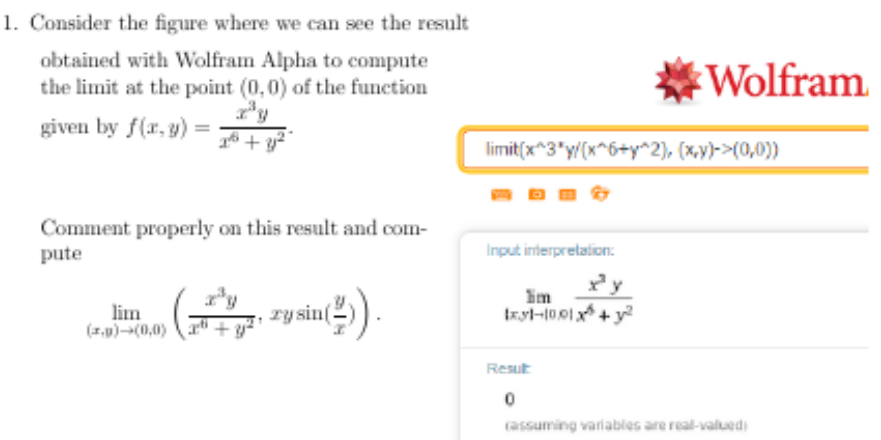

Fig. 2. Testing Wolfram Alpha in computing the limit of function in a given point.

For a number of 164 students, 118 used their knowledge of the limit concept and theorems to refuse this response (in fact, the proposed limit does not exist in the given point) and also some of them were able to give a correct answer and predict a reason for the algorithmic failure. Unfortunately, about $28 \%$ of the students surveyed trusted blindly in the result, invoking, in some cases, the impossibility of wrong answer and forcing a proof of the desired result.

As a remark, it is important to mention that at the time this paper is being written the failure of Wolfram Alpha in this particular case is solved; however, in other related cases the error still remains. For instance, this system also gives the value 0 for $\lim _{(x, y) \mapsto(0,0)} \frac{x^{2} y}{x^{2}+y^{5}}$ (see figure 3 ) although such limit does not exist as well.

\section{WolframAl}

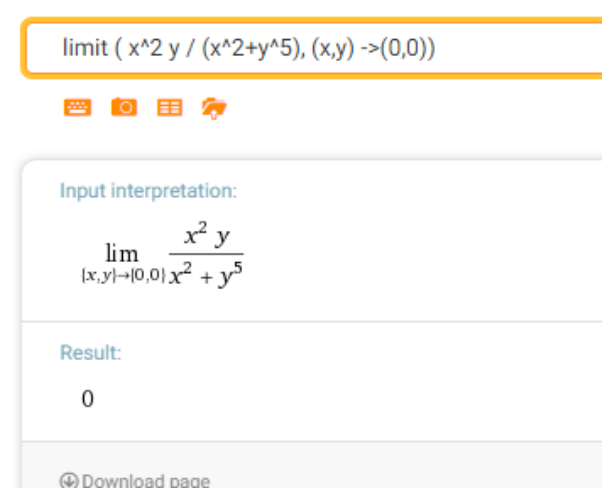

Fig. 3. Another Wolfram Alpha failure in computing the limit of function at a given point.

This reference together with example 1 in the previous section show the relevance of mathematical knowledge and critical thinking in problem solving when using technology.

\section{LEARNING AND ASSESSMENT}

Learning is the activity or process of gaining knowledge or skill by studying, practicing, being taught, or experiencing something: the activity of someone who learns. Assessment is the act of making a judgment about something: the act of assessing something [14].

These two words always come together when the concern is education. Nowadays teachers and students can make use of the internet to support learning activities as well as evaluation. In YouTube there are channels specially dedicated to education one can subscribe according to the grades desired and Khan Academy is the most well-known on line platform delivering resources, in the form of quizzes, assignments, exercises, etc., fostering self-assessment.

\section{A. Technological on line platforms}

Learning mathematics requires students to work in an independent way which is particularly challenging for such a subject like Calculus. Advancements in technology, taking the student as the focus of his own learning, led to a change of paradigm in education. For example, worked out examples constitute an important element in textbook presentations of new concepts and procedures. These examples can be shown to contain enough information about the procedures to permit diligent students to learn them without additional instruction, a fact many good students appear to know and exploit. Presumably, the students infer from the examples the essential procedures and then internalize them so that they can apply these procedures to new problems [8].

Despite the discussion this procedure may cause, we developed two technological platforms consisting of an interactive feedback and assessment tool based on parameterized math exercises, and we use it to motivate student's independent study in some curricular units of calculus courses. 
Both platforms can operate individually or they can be used as complement to one another. One of these platforms is a Web application, Siacua (which stands for Interactive System form Learning by Computer in Portuguese)] [4] which implements a Bayesian user model, with an algorithm for knowledge propagation. Each student has an associated Bayesian network and each node of this network represents a topic of the course lectured: in the case described in this paper, Calculus I, Calculus II and Calculus III. Before starting to answer questions, the student's Bayesian network only contains concepts; these concepts constitute the conceptual map of the course. Each time the student answers a question, a new node is added to the network, called an evidence node, connected with the concepts it involves, and the student's knowledge spreads. Using Siacua students can see their progress, which is shown in the form of progress bars. The Bayesian network associated with the student is used to update all progress bars whenever an evidence is provided by answering a question.

Students have immediate feedback about their performance, in other words, they know if the answer given is right or wrong, in the latter they can see the detailed solution, and have information about their general progress in the course, given by the progress bars, as illustrated in Figure 4.

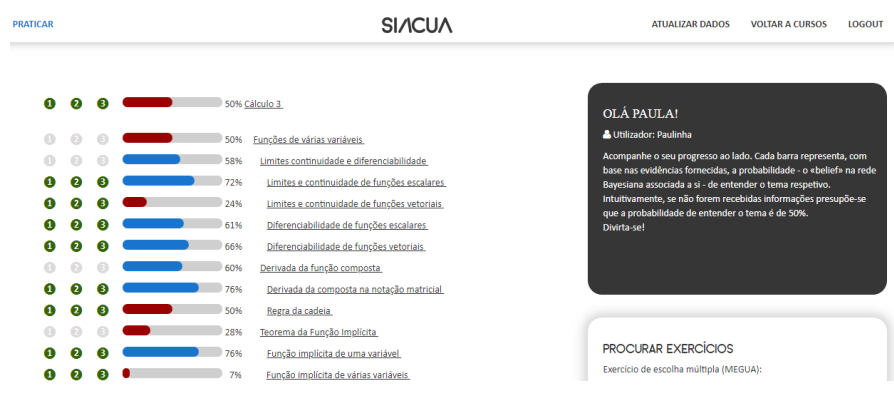

Fig. 4. Siacua: table of progress.

On the other hand we use another Web Application, PmatE, to implement self-assessment. Teachers build evaluation tests using random questions (see [6], [7] for details) of one or both platforms about some chosen topic.

The main goal of this platform is to support teaching, making it possible to: manage the groups involved; elaborate tests; assess students performance; analyse results and perform other management tasks. The developed programs are thus a tool to support education, evaluation and learning [13].

The interaction with this platform is minimal, consisting only of answering true or false questions. After submitting the test students know immediately their grades and can visualize the test submitted where the wrong and correct answers are indicated, and so can teachers (see figure 5).

The ease on extracting results to an excel file directly from the platform facilitates the task of correcting hundreds of tests.

\section{RESULTS AND DISCUSSION}

On the second semester of the academic year 2016/2017 there were 394 students enrolled in Calculus II (degrees

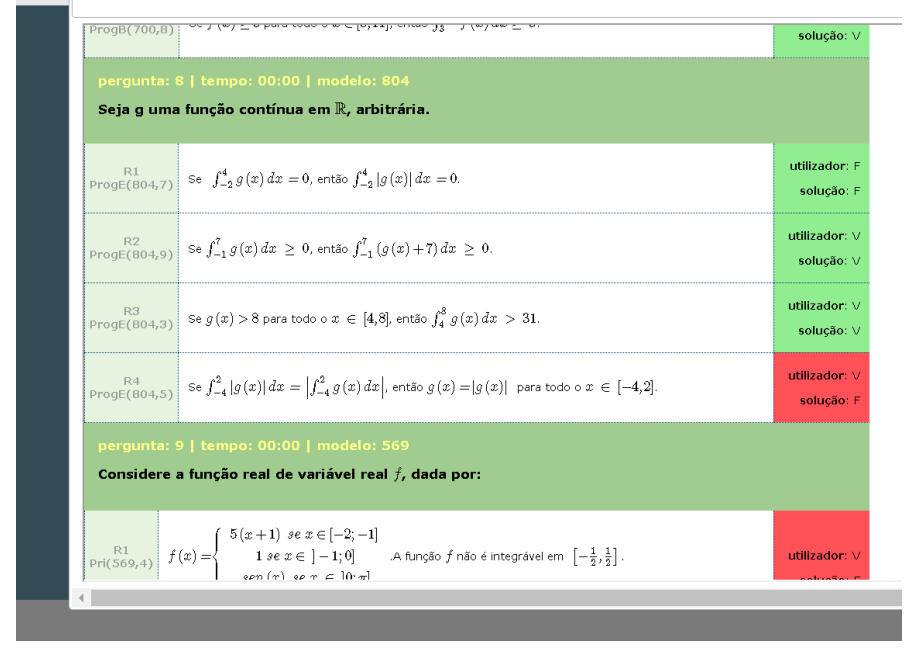

Fig. 5. An excerpt of a test in PmatE.

in Chemical Engineering, Biochemistry, Biotechnology, Materials Engineering, Environmental Engineering and Marine Sciences) distributed by 9 classes taught by 4 teachers. From these 394 students only 325 were enrolled in some class and the average of attendance was 273 . In this semester there was an average of 27 lectures, 6 of which were flipped on chosen topics, not too difficult for students to learn by themselves. In the last 20 minutes of the lecture each student answered individually to a question (class question) on the subject of that specific topic.

To support autonomous study, a detailed text was available on moodle and the online platform with worked-out exercises, described in section IV.

By the end of the semester an inquiry to evaluate the functioning of the curricular unit was distributed to all classes. The inquiry consisted of only four items:

- Identify the weaknesses of the curricular unit operation.

- Identify the strengths of the curricular unit operation.

- Estimate the time spent per week studying this curricular unit.

- Give suggestions (either for the teacher or for the curricular unit operation).

There was a total of 224 respondents, listing 202 weaknesses, 386 strengths and 147 suggestions. In particular, on the subject flipped classroom, 14 students identified them as a strength on the curricular unit, 2 wanted the number of these classes to increase, against 5 that wanted it to decrease. In spite of the small number of students who see flipped classroom as a strength, 68 students considered the assessment tests on the end of these classes also a strong point.

The respondents mentioned 26 different weaknesses and 17 strengths that we grouped in six classes: syllabus, assessment, classes, materials (educational), teacher and online platforms, making it easier to compare both, as illustrated on figure 6 .

As weaknesses, students referred the following ${ }^{1}$ :

\footnotetext{
${ }^{1}$ In brackets is the number of students who pointed that weakness/strength.
} 


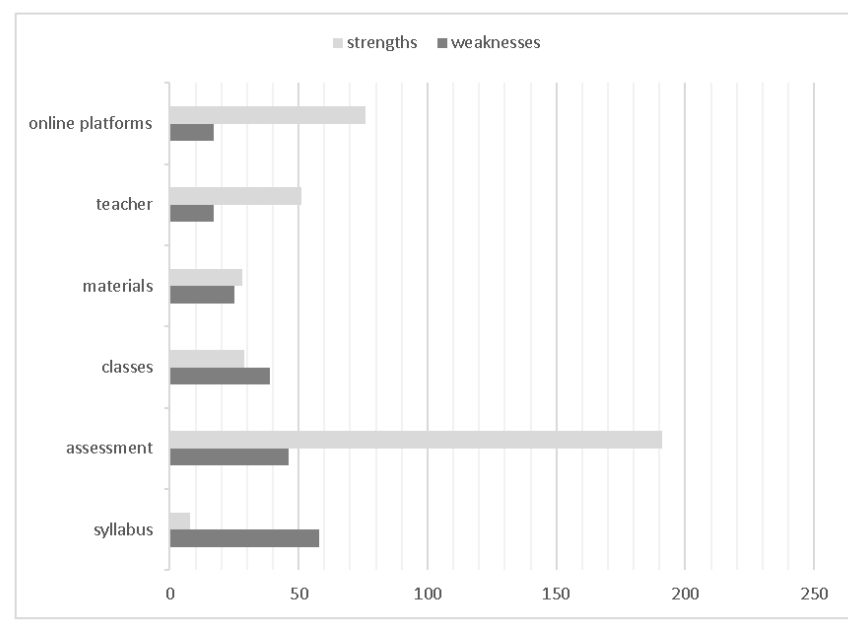

Fig. 6. Weaknesses and strengths pointed out by the students.

- Syllabus: too long (33) or difficult (9) curriculum, 3 students considered it old fashioned and 13 students think it is not articulated with their courses.

- Assessment: too many class questions and homework (19), 12 students consider the tests/exams difficult, unbalanced distribution in the tests of the subjects lectured(6), the class questions shouldn't be on the topics of the flipped classroom (4) and the different levels of difficulty between class questions (2).

- Classes: too theoretical classes (13), few classes per week (1), classes too similar to slides (1), over-crowded classes (1), too many flipped classrooms (3), non-ventilated rooms (3), absences regimen (5), the timetable (7).

- Materials: few worked-out exercises (19), text of support to the curricular unit not explicit enough (4), no videos or animated gifs (1), bad slides (1).

- Teachers: teacher (6), changing the teacher during the semester (11).

- Online platforms: using on-line platforms for assessment and exercises available not similar to those on assessment tests (17)

Regarding strengths, the answers were distributed as follows:

- Syllabus: appropriate syllabus (8).

- Assessment: various assessment moments keep an up-todate study (88), existence of class questions(68), existence of homework (35).

- Classes: flipped classrooms (14), classes with many exercises (5), many illustrative examples (5), summaries in the blackboard (1), dynamic classes (1).

- Materials: text of support to the curricular unit (14), many resources available (14).

- Teacher: the teacher himself as a strength (51).

- On-line platforms: SIACUA with detailed answers (47) and SIACUA and PmatE together (29).

For being very scattered we will only mention the suggestions that gathered eight or more answers:

- Keep it as it is (27).

- Provide more worksheets with solved exercises as well as the detailed resolution of tests and exams from previous years (24).

- Less theoretical classes (17).

- Ignore the worst grade obtained in the class questions (11).

- Reduce the number of class questions and homeworks (8).

The analysis of this data leads us to look for strategies focused on the active work, practice and autonomous work of the student, even though it is difficult to do this when it comes to mathematics.

\section{CONCLUSION}

It seems to be imperative to adapt the methodologies on teaching Calculus to Engineering Courses. The development of technologies and technological systems of computation, some of them devoted to education, as well as social evolution, forces teachers and students to use them. However, all the examples highlighted in this paper clarify the idea that this change cannot neglect a strong background in mathematical knowledge and active criticism in problem solving.

\section{ACKNOWLEDGMENT}

The research of Paula Carvalho and Paula Oliveira are supported by the Portuguese Foundation for Science and Technology ("FCT-Fundação para a Ciência e a Tecnologia"), through the CIDMA - Center for Research and Development in Mathematics and Applications, within project UID/MAT/04106/2013

\section{REFERENCES}

[1] D. Harris, L. Black, P. Hernandez-Martinez, B. Pepin and J. Williams, \& TransMaths Team (2015). "Mathematics and its value for Engineering students: what are the implications for teaching?," International Journal of Mathematical Education in Science and Technology, 46(3), pp. 321336,2015

[2] J. Ellis, M. L. Kelton and C. Rasmussen, "Student perceptions of pedagogy and associated persistence in calculus", ZDM, 46, n.4, pp.661$673,2014$.

[3] (2018, Feb.) Wolfram Alpha: Computational Knowledge Engine, [Online]. Available: http://www.wolfram.com/

[4] (2018, Feb.) SIACUA, Sistema Interativo de Aprendizagem por Computador Universidade de Aveiro. [Online]. Available: http://siacua.web.ua.pt/

[5] (2018, Feb.) PmatE, Projecto Matemtica Ensino Universidade de Aveiro. [Online]. Available: http://pmate.ua.pt/

[6] L. Descalço and P. Carvalho, "Using parameterized calculus questions for learning and assessment", Information Systems and Technologies (CISTI), 10th Iberian Conference on Information Systems and Technologies (CISTI), 2015.

[7] L. Descalço, P. Carvalho, J. P. Cruz, P. Oliveira, and D. Seabra, "Using bayesian networks and parameterized questions in independent study", EDULEARN15 Proceedings, 7th International Conference on Education and New Learning Technologies, Barcelona, pp.3361-3368, 2015.

[8] X. Zhu and H. Simon, "Learning mathematics from examples and by doing", Cognition and instruction, Ed. Taylor \& Francis, vol. 4, n. 3 , pp.137-166, 1987.

[9] Board on Engineering Education, National Research Council, "Engineering Education: Designing an Adaptive System”, National Academies Press, ISBN: 0-309-52051-7, 1995. 
[10] (2018, Feb.) "I was wondering what types of mathematics an engineer uses on a day to day basis. For example, do they use calculus concepts daily?", [Online]. Available: http://tryengineering.org/ask-expert/

[11] (2018, Feb.) H. B. Marcoux, "What is the importance of maths in engineering?", [Online]. Available: https://www.quora.com/

[12] P. Baepler, J.D. Walker and M. Driessen, "It's not about seat time: Blending, flipping, and efficiency in active learning classrooms", Computers \& Education, v. 78, p. 227-236, 2014.

[13] S. Pais, I. Cabrita and A. B. Anjo, "The Use of Mathematics Education Project in the Learning of Mathematical Subjects at University Level", International Journal of Education, Vol. 3, No. 1: E4. ISSN 1948-5476, 2011.

[14] $(2018, \quad$ Feb.) Merriam-Webster, [Online]. Available: http://www.learnersdictionary.com/ 